\title{
OSCILLATION OF SYSTEMS OF CERTAIN NEUTRAL DELAY PARABOLIC DIFFERENTIAL EQUATIONS ${ }^{1}$
}

\author{
WEI NIAN LI \\ Shanghai Jiaotong University \\ Department of Mathematics \\ Shandong 200240, PR of China \\ BAO TONG CUI \\ Binzhou Normal College \\ Department of Mathematics \\ Shandong 256604, PR of China \\ LOKENATH DEBNATH \\ University of Texas-Pan American \\ Department of Mathematics \\ Edinburg, TX 78539 USA
}

(Received February, 2002; Revised June, 2002)

\begin{abstract}
Sufficient conditions are established for the oscillation of systems of neutral delay parabolic differential equations. These results are illustrated by some examples.

Key words: Oscillation, System, Neutral Delay, Parabolic Differential Equation.

AMS subject classifications: 35B05, 35R10.
\end{abstract}

\section{Introduction}

During the past decade, considerable attention has been given to boundary value problems and initial value problems for partial differential equations with piecewise constant delay by several authors including Wiener and Debnath [9-11]. In all of these papers, the major focus was to investigate the influence of certain piecewise constant time delays, continuous time delays and discontinuous time delays on the solutions of partial differential equations. These results have also been extended to equations with positive definite operators in Hilbert spaces. A class of initial value problems for partial differential equations with piecewise constant argument (EPCA) in partial derivatives. A class of loaded partial differential equations that arise in solving certain inverse problems has been studied within the general framework with piecewise constant delay. An abstract Cauchy problem for partial differential equations with time delays

${ }^{1}$ This work is supported by the National Science Foundation of Shandong Province, China (Y2001A03). 
in a Banach space has also been examined by Wiener and Debnath [11]. Subsequently, Wiener and Debnath [12] have studied boundary value problems for the diffusion equation with piecewise continuous time delay. This study included boundary value problems for three types of equations: delayed, alternately advanced, and retarded type and most importantly, equations of neutral type. These equation included loaded and impulsive equations as special cases and hence their importance arises in control theory and in certain biomedical models. Recently, Wiener and Heller [13] have made a detailed study of diffusion equations of neutral type with piecewise constant time delay. This study reveals many interesting features including oscillatory and periodic properties of the solutions. On the other hand, Wiener and Debnath [12] have examined the oscillatory properties of the wave equation with discontinuous time delay.

In addition, several authors including Mishev and Bainov [8], Fu and Zhuang [4], Cui et al [2], Bainov et al [1], Li and Cui [6], Debnath and Li [3] have studied the oscillation problems for the partial differential equations of different types. Very recently, Li and Debnath [7] have investigated the theory of oscillations of a system of hyperbolic partial differential equations with continuous distributed deviating arguments. They have obtained sufficient conditions for the oscillation of the system of delay hyperbolic partial differential equations with examples. In spite of the above studies, hardly any attention was given to the problem of oscillation of a system of certain neutral delay parabolic differential equations. The main objective of this paper is to study this problem. Sufficient condition are proved for the oscillation of systems of neutral delay parabolic equations with some examples.

\section{Formulation of the Problem}

In this paper, we study the oscillation of systems of neutral delay parabolic differential equations of the form

$$
\begin{gathered}
\frac{\partial}{\partial t}\left[u_{i}(x, t)-\sum_{s=1}^{r} \lambda_{s}(t) u_{i}\left(x, t-\rho_{s}\right)\right]-a_{i}(t) \Delta u_{i}(x, t)+\sum_{k=1}^{m} \sum_{j=1}^{d} a_{i k j}(t) \Delta u_{k}\left(x, t-\tau_{j}\right) \\
-p_{i}(x, t) u_{i}(x, t)-\sum_{k=1}^{m} \sum_{h=1}^{l} \int_{a}^{b} q_{i k h}(x, t, \xi) u_{k}\left(x, g_{h}(t, \xi)\right) d \sigma(\xi) \\
(x, t) \in \Omega \times[0, \infty) \equiv G, i=1,2, \ldots, m
\end{gathered}
$$

where $\Omega$ is a bounded domain in $R^{n}$ with a piecewise smooth boundary $\partial \Omega$,

$\Delta u_{i}(x, t)=\sum_{r=1}^{n} \frac{\partial^{2} u_{i}(x, t)}{\partial x_{r}^{2}}, i=1,2, \ldots, m$, and the integral in (2.1) is the Riemann-Stieltjes integral.

In this paper, we always suppose that the following conditions hold:

(H1) $a_{i} \in C([0, \infty) ;[0, \infty)), a_{i k j} \in C([0, \infty) ; R), a_{i i j}(t)>0$, and

$$
\begin{aligned}
& A_{j}(t) \underset{1 \leq i \leq m}{=}\left\{a_{i i j}(t)-\sum_{k=1, k \neq i}^{m}\left|a_{k i j}(t)\right|\right\}>0, i=1,2, \ldots, m ; j=1,2, \ldots, d ; \\
& \text { (H2) } \lambda_{s} \in C^{1}([0, \infty) ;[0, \infty)), s=1,2, \ldots, r
\end{aligned}
$$


(H3) $p_{i} \in C(\bar{G} ;[0, \infty)), p_{i}(t)=\inf _{x \in \bar{\Omega}} p_{i}(x, t), p(t)=\inf _{1 \leq i \leq m}\left\{p_{i}(t)\right\}, i=1,2, \ldots, m$;

(H4) $q_{i k h} \in C(\bar{G} \times[a, b] ; R), q_{i i h}(x, t, \xi)>0$, and

$$
\begin{gathered}
q_{i i h}(t, \xi)=\inf _{x \in \bar{\Omega}} q_{i i h}(x, t, \xi), \bar{q}_{i k h}(t, \xi)=\sup _{x \in \bar{\Omega}}\left|q_{i k h}(x, t, \xi)\right|, \\
Q_{h}(t, \xi)=\min _{1 \leq i \leq m}\left\{q_{i i h}(t, \xi)-\sum_{k=1, k \neq i}^{m} \bar{q}_{k i h}(t, \xi)\right\} \geq 0,
\end{gathered}
$$

where $i, k=1,2, \ldots, m ; h=1,2, \ldots, l$;

(H5) $g_{h} \in C([0, \infty) \times[a, b] ; R), g_{h}(t, \xi) \leq t, \xi \in[a, b]$ and $g_{h}(t, \xi)$ is a nondecreasing function with respect to $t$ and $\xi$, respectively,

$$
\lim _{t \rightarrow \infty} \min _{\xi \in[a, b]}\left\{g_{h}(t, \xi)\right\}=\infty, h=1,2, \ldots, l ;
$$

(H6) $\sigma \in([a, b] ; R)$ and $\sigma(\xi)$ is nondecreasing in $\xi$;

(H7) $\rho_{s}, \tau_{j}$ are positive constants, $s=1,2, \ldots, r ; j=1,2, \ldots, d$.

We consider two kinds of boundary conditions:

$$
\frac{\partial u_{i}(x, t)}{\partial N}+f_{i}(x, t) u_{i}(x, t)=0,(x, t) \in \partial \Omega \times[0, \infty), i=1,2, \ldots, m,
$$

where $N$ is the unit exterior normal vector to $\partial \Omega$ and $f_{i}(x, t)$ is a nonnegative continuous function on $\partial \Omega \times[0, \infty), i=1,2, \ldots, m$, and

$$
u_{i}(x, t)=0,(x, t) \in \partial \Omega \times[0, \infty), i=1,2, \ldots, m .
$$

Definition 2.1: The vector function $u(x, t)=\left\{u_{1}(x, t), u_{2}(x, t), \ldots, u_{m}(x, t)\right\}^{T}$ is said to be a solution of the problem (2.1), (2.2) (or (2.1), (2.3)) if it satisfies (2.1) in $G=$ $\Omega \times[0, \infty)$ and boundary condition (2.2) (or (2.3)).

Definition 2.2: The vector solution $u(x, t)=\left\{u_{1}(x, t), u_{2}(x, t), \ldots, u_{m}(x, t)\right\}^{T}$ of the problem (2.1), (2.2) (or (2.1), (2.3)) is said to be oscillatory in the domain $G=\Omega \times[0, \infty)$ if at least one of its nontrivial component is oscillatory in $G$. Otherwise, the vector solution $u(x, t)$ is said to be nonoscillatory.

\section{Oscillation of the Problem (2.1), (2.2)}

Theorem 3.1: If the neutral differential inequality

$\left[V(t)-\sum_{s=1}^{r} \lambda_{s}(t) V\left(t-\rho_{s}\right)\right]^{\prime}+p(t) V(t)+\sum_{h=1}^{l} \int_{a}^{b} Q_{h}(t, \xi) V\left(g_{h}(t, \xi)\right) d \sigma(\xi) \leq 0$,

has no eventually positive solution, then every solution of the problem (2.1), (2.2) is oscillatory in $G$.

Proof: Suppose to the contrary that there is a nonoscillatory solution $u(x, t)=$ $\left\{u_{1}(x, t), u_{2}(x, t), \ldots, u_{m}(x, t)\right\}^{T}$ of the problem (2.1), (2.2). We assume that $\left|u_{i}(x, t)\right|>0$ 
for $t \geq t_{0} \geq 0, i=1,2, \ldots, m$. Let $\delta_{i}=\operatorname{sgn} u_{i}(x, t), Z_{i}(x, t)=\delta_{i} u_{i}(x, t)$, then $Z_{i}(x, t)>0$, $(x, t) \in \Omega \times\left[t_{0}, \infty\right), i=1,2, \ldots, m$. From (H5) and (H7) there exists a number $t_{1} \geq t_{0}$ such that $Z_{i}(x, t)>0, Z_{i}\left(x, t-\rho_{s}\right)>0, Z_{i}\left(x, t-\tau_{j}\right)>0$ and $Z_{i}\left(x, g_{h}(t, \xi)\right)>0$ in $\Omega \times\left[t_{1}, \infty\right)$, $i=1,2, \ldots, m ; s=1,2, \ldots, r ; j=1,2, \ldots, d ; h=1,2, \ldots, l$.

Integrating (2.1) with respect to $x$ over the domain $\Omega$, we have

$$
\begin{gathered}
\frac{d}{d t}\left[\int_{\Omega} u_{i}(x, t) d x-\sum_{s=1}^{r} \lambda_{s}(t) \int_{\Omega} u_{i}\left(x, t-\rho_{s}\right) d x\right]=a_{i}(t) \int_{\Omega} \Delta u_{i}(x, t) d x \\
\quad+\sum_{k=1}^{m} \sum_{j=1}^{d} a_{i k j}(t) \int_{\Omega} \Delta u_{k}\left(x, t-\tau_{j}\right) d x-\int_{\Omega} p_{i}(x, t) u_{i}(x, t) d x \\
-\sum_{k=1}^{m} \sum_{h=1}^{l} \int_{\Omega} \int_{a}^{b} q_{i k h}(x, t, \xi) u_{k}\left(x, g_{h}(t, \xi)\right) d \sigma(\xi) d x, t \geq t_{1}, i=1,2, \ldots, m,
\end{gathered}
$$

where $t \geq t_{1}, i=1,2, \ldots, m$.

It is easy to see that

$$
\begin{aligned}
& \int_{\Omega} \int_{a}^{b} q_{i k h}(x, t, \xi) u_{k}\left(x, g_{h}(t, \xi)\right) d \sigma(\xi) d x \\
& =\int_{a}^{b} \int_{\Omega}^{b} q_{i k h}(x, t, \xi) u_{k}\left(x, g_{h}(t, \xi)\right) d x d \sigma(\xi) .
\end{aligned}
$$

Therefore,

$$
\begin{gathered}
\frac{d}{d t}\left[\int_{\Omega} Z_{i}(x, t) d x-\sum_{s=1}^{r} \lambda_{s}(t) \int_{\Omega} Z_{i}\left(x, t-\rho_{s}\right) d x\right]=a_{i}(t) \int_{\Omega} \Delta Z_{i}(x, t) d x \\
+\sum_{j=1}^{d} a_{i i j}(t) \int_{\Omega} \Delta Z_{i}\left(x, t-\tau_{j}\right) d x+\sum_{k=1, k \neq i}^{m} \sum_{j=1}^{d} a_{i k j}(t) \frac{\delta_{k}}{\delta_{i}} \int_{\Omega} \Delta Z_{k}\left(x, t-\tau_{j}\right) d x \\
-\int_{\Omega} p_{i}(x, t) Z_{i}(x, t) d x-\sum_{h=1}^{l} \int_{a}^{b} \int_{\Omega}^{b} q_{i i h}(x, t, \xi) Z_{i}\left(x, g_{h}(t, \xi)\right) d x d \sigma(\xi) \\
-\sum_{h=1}^{l} \sum_{k=1, k \neq i}^{m} \frac{\delta_{k}}{\delta_{i}} \int_{a}^{b} \int_{\Omega} q_{i k h}(x, t, \xi) Z_{k}\left(x, g_{h}(t, \xi)\right) d x d \sigma(\xi),
\end{gathered}
$$

where $t \geq t_{1}, i=1,2, \ldots, m$.

The Green's formula and (2.2) yield 
and

$$
\int_{\Omega} \Delta Z_{i}(x, t) d x=\int_{\partial \Omega} \frac{\partial Z_{i}(x, t)}{\partial N} d S=-\int_{\partial \Omega} f_{i}(x, t) Z_{i}(x, t) d S \leq 0,
$$

$$
\begin{gathered}
\int_{\Omega} \Delta Z_{k}\left(x, t-\tau_{j}\right) d x=\int_{\partial \Omega} \frac{\partial Z_{k}\left(x, t-\tau_{j}\right)}{\partial N} d S \\
=-\int_{\partial \Omega} f_{k}\left(x, t-\tau_{j}\right) Z_{k}\left(x, t-\tau_{j}\right) d S,
\end{gathered}
$$

where $t \geq t_{1}, i, k=1,2, \ldots, m ; j=1,2, \ldots, d$, and $d S$ is the surface element on $\partial \Omega$.

Combining (3.4)-(3.6), we get

$$
\begin{gathered}
\frac{d}{d t}\left[\int_{\Omega} Z_{i}(x, t) d x-\sum_{s=1}^{r} \lambda_{s}(t) \int_{\Omega} Z_{i}\left(x, t-\rho_{s}\right) d x\right] \\
\leq-\sum_{j=1}^{d} a_{i i j}(t) \int_{\partial \Omega} f_{i}\left(x, t-\tau_{j}\right) Z_{i}\left(x, t-\tau_{j}\right) d S \\
+\sum_{j=1}^{d} \sum_{k=1, k \neq i}^{m}\left|a_{i k j}(t)\right| \int_{\partial \Omega} g_{k}\left(x, t-\tau_{j}\right) Z_{k}\left(x, t-\tau_{j}\right) d S-p_{i}(t) \int_{\Omega} Z_{i}(x, t) d x \\
+\sum_{h=1}^{l} \sum_{h=1}^{l} \int_{a}^{m} q_{i i h}^{b}(t, \xi) \int_{\Omega} Z_{i}\left(x, g_{h}(t, \xi)\right) d x d \sigma(\xi) \\
\int_{a}^{b} \bar{q}(t, \xi) \int_{\Omega} Z_{k}\left(x, g_{h}(t, \xi)\right) d x d \sigma(\xi),
\end{gathered}
$$

where $t \geq t_{1}, i=1,2, \ldots, m$.

Setting

$$
V_{i}(t)=\int_{\Omega} Z_{i}(x, t) d x, W_{i}(t)=\int_{\partial \Omega} f_{i}(x, t) Z_{i}(x, t) d S, t \geq t_{1}, i=1,2, \ldots, m,
$$

we obtain

$$
\begin{gathered}
{\left[V_{i}(t)-\sum_{s=1}^{r} \lambda_{s}(t) V_{i}\left(t-\rho_{s}\right)\right]^{\prime}+\sum_{j=1}^{d} a_{i i j}(t) W_{i}\left(t-\tau_{j}\right)} \\
\quad-\sum_{j=1}^{d} \sum_{k=1, k \neq i}^{m}\left|a_{i k j}(t)\right| W_{k}\left(t-\tau_{j}\right)+p_{i}(t) V_{i}(t)
\end{gathered}
$$




$$
\begin{gathered}
+\sum_{h=1}^{l} \int_{a}^{b} q_{i i h}(t, \xi) V_{i}\left(g_{h}(t, \xi)\right) d \sigma(\xi) \\
-\sum_{h=1}^{l} \sum_{k=1, k \neq 1}^{m} \int_{a}^{b} \bar{q}_{i k h}(t, \xi) V_{k}\left(g_{h}(t, \xi)\right) d \sigma(\xi) \leq 0,
\end{gathered}
$$

where $t \geq t_{1}, i=1,2, \ldots m$.

Let $V(t)=\sum_{i=1}^{m} V_{i}(t), W(t)=\sum_{i=1}^{m} W_{i}(t)$ for $t \geq t_{1}$. It follows from (3.8) that

$$
\begin{gathered}
{\left[V(t)-\sum_{s=1}^{r} \lambda_{s}(t) V\left(t-\rho_{s}\right)\right]^{\prime}+\sum_{j=1}^{d}\left\{\sum_{i=1}^{m}\left[a_{i i j}(t) W_{i}\left(t-\tau_{j}\right)-\sum_{k=1, k \neq i}^{m}\left|a_{i k j}(t)\right| W_{k}\left(t-\tau_{j}\right)\right]\right\}} \\
+p(t) V(t)+\sum_{h=1}^{l}\left\{\sum _ { i = 1 } ^ { m } \int _ { a } ^ { b } \left[q_{i i h}(t, \xi) V_{i}\left(t_{h}(t, \xi)\right) d \sigma(\xi)\right.\right. \\
\left.\left.\quad-\sum_{k=1, k \neq i}^{m} \int_{a}^{b} \bar{q}_{i k h}(t, \xi) V_{k}\left(g_{h}(t, \xi)\right) d \sigma(\xi)\right]\right\} \leq 0, t \geq t_{1}
\end{gathered}
$$

Noting that

$$
\begin{aligned}
& \sum_{i=1}^{m} \int_{a}^{b}\left[q_{i i h}(t, \xi) V_{i}\left(g_{h}(t, \xi)\right)-\sum_{k=1, k \neq i}^{m} \bar{q}_{i k h}(t, \xi) V_{k}\left(g_{h}(t, \xi)\right)\right] d \sigma(\xi) \\
& =\int_{a}^{b}\left[q_{11 h}(t, \xi) V_{1}\left(g_{h}(t, \xi)\right)-\sum_{k=1, k \neq 1}^{m} \bar{q}_{1 k h}(t, \xi) V_{k}\left(g_{h}(t, \xi)\right)\right] d \sigma(\xi) \\
& +\int_{a}^{b}\left[q_{22 h}(t, \xi) V_{2}\left(g_{h}(t, \xi)\right)-\sum_{k=1, k \neq 2}^{m} \bar{q}_{2 k h}(t, \xi) V_{k}\left(g_{h}(t, \xi)\right)\right] d \sigma(\xi) \\
& +\int_{a}^{b}\left[q_{m m h}(t, \xi) V_{m}\left(g_{h}(t, \xi)\right)-\sum_{k=1, k \neq m}^{m} \bar{q}_{m k h}(t, \xi) V_{k}\left(g_{h}(t, \xi)\right)\right] d \sigma(\xi) \\
& =\int_{a}^{b}\left[q_{11 h}(t, \xi)-\sum_{k=1, k \neq 1}^{m} \bar{q}_{k 1 h}(t, \xi)\right] V_{1}\left(g_{h}(t, \xi)\right) d \sigma(\xi)
\end{aligned}
$$




$$
\begin{gathered}
+\int_{a}^{b}\left[q_{22 h}(t, \xi)-\sum_{k=1, k \neq 2}^{m} \bar{q}_{k 2 h}(t, \xi)\right] V_{2}\left(g_{h}(t, \xi)\right) d \sigma(\xi) \\
+\ldots \\
\left.+\int_{a}^{b} q_{m m h}(t, \xi) V_{m}\left(g_{h}(t, \xi)\right)-\sum_{k=1, k \neq m}^{m} \bar{q}_{k m h}(t, \xi) V_{m}\left(g_{h}(t, \xi)\right)\right] d \sigma(\xi) \\
\geq \int_{a}^{b} \min _{1 \leq i \leq m}\left\{q_{i i h}(t, \xi)-\sum_{k=1, k \neq i}^{m} \bar{q}_{k i h}(t, \xi)\right\} \sum_{i=1}^{m} V_{i}\left(g_{h}(t, \xi)\right) d \sigma(\xi) \\
=\int_{a}^{b} Q_{h}(t, \xi) V\left(g_{h}(t, \xi)\right) d \sigma(\xi), t \geq t_{1}, h=1,2, \ldots, l .
\end{gathered}
$$

Similarly,

$$
\begin{gathered}
\sum_{i=1}^{m}\left[a_{i i j}(t) W_{i}\left(t-\tau_{j}\right)-\sum_{k=1, k i}^{m}\left|a_{i k j}(t)\right| W_{k}\left(t-\tau_{j}\right)\right] \\
\geq \min _{1 \leq i \leq m}\left[a_{i i j}(t)-\sum_{k=1, k i}^{m}\left|a_{k i j}\right|\right] \sum_{i=1}^{m} W_{i}\left(t-\tau_{j}\right) \\
=A_{j}(t) W\left(t-\tau_{j}\right), t \geq t_{1}, j=1,2, \ldots, d .
\end{gathered}
$$

Then, from (3.9), we have

$$
\begin{gathered}
{\left[V(t)-\sum_{s=1}^{r} \lambda_{s}(t) V\left(t-\rho_{s}\right)\right]^{\prime}+\sum_{j=1}^{d} A_{j}(t) W\left(t-\tau_{j}\right)+p(t) V(t)} \\
\quad+\sum_{h=1}^{l} \int_{a}^{b} Q_{h}(t, \xi) V\left(g_{h}(t, \xi)\right) d \sigma(\xi) \leq 0, t \geq t_{1} .
\end{gathered}
$$

It is easy to see that

$$
W\left(t-\tau_{j}\right)=\sum_{i=1}^{m} W_{i}\left(t-\tau_{j}\right) \geq 0, t \geq t_{1}, j=1,2, \ldots, d
$$

Therefore, 


$$
\begin{gathered}
{\left[V(t)-\sum_{s=1}^{r} \lambda_{s}(t) V\left(t-\rho_{s}\right)\right]^{\prime}+p(t) V(t)} \\
+\sum_{h=1}^{l} \int_{a}^{b} Q_{h}(t, \xi) V\left(g_{h}(t, \xi)\right) d \sigma(\xi) \leq 0, \quad t \geq t_{1},
\end{gathered}
$$

which contradicts the assumption that (3.1) has no eventually positive solution. This completes the proof.

Theorem 3.2: Suppose there exists some $h_{0} \in\{1,2, \ldots, l\}$ such that

(B1) there exists a function $\eta_{h_{0}} \in C([0, \infty) \times[a, b] ;(0, \infty))$ such that

$$
\eta_{h_{0}}\left(\eta_{h_{0}}(t, \xi), \xi\right)=g_{h_{0}}(t, \xi)
$$

$\eta_{h_{0}}(t, \xi)$ is a nondecreasing function with respect to $t$ and $\xi$, and

$$
t \geq \eta_{h_{0}}(t, \xi) \geq g_{h_{0}}(t, \xi), \lim _{t \rightarrow \infty} \min _{\xi \in[a, b]} \eta_{h_{0}}(t, \xi)=\infty ;
$$

(B2) $\liminf _{t \rightarrow \infty} \int_{g_{h_{0}}(t, b)}^{t} \int_{a}^{b} Q_{h_{0}}(s, \xi) d \sigma(\xi) d s>\frac{1}{e}$;

(B3) $\liminf f_{t \rightarrow \infty} \int_{\eta_{h_{0}}(t, b)}^{t} \int_{a}^{b} Q_{h_{0}}(s, \xi) d \sigma(\xi) d s>0$;

(B4) $\lim _{t \rightarrow \infty} \sum_{s=1}^{r} \lambda_{s}(t)=\lambda$ and $0<\lambda<1$;

(B5) there exists a constant $m>0$ such that $Q_{h_{0}}(t, \xi) \geq m,(t, \xi) \in[0, \infty) \times[a, b]$.

Then every solution of the problem (2.1), (2.2) is oscillatory in $G$.

Proof: We prove that inequality (3.1) has no eventually positive solution if the conditions of Theorem 3.2 hold. Suppose $V(T)$ is an eventually positive solution of inequality (3.1). Then there exists a number $t_{1} \geq 0$ such that $V\left(g_{h}(t, \xi)\right)>0, h=1,2, \ldots, l$, for $t \geq t_{1}$. Thus we have

$$
\begin{aligned}
& {\left[V(t)-\sum_{s=1}^{r} \lambda_{s}(t) V\left(t-\rho_{s}\right)\right]^{\prime}+p(t) V(t) } \\
+ & \int_{a}^{b} Q_{h_{0}}(t, \xi) V\left(g_{h_{0}}(t, \xi)\right) d \sigma(\xi) \leq 0, \quad t \geq t_{1} .
\end{aligned}
$$

By Theorem 2 of Fu and Zhang [4], we obtain that inequality (3.11) has no eventually solution, which contradicts the fact that $V(t)>0$ is a solution of inequality (3.11).

The proof of the following theorem is similar to that of Theorem 3.2 by using Theorem 3 of Fu and Zhang [4].

Theorem 3.3: Suppose that conditions (B1), (B2), (B3) and (B5) hold. If

(B4) $\sum_{s=1}^{r} \lambda_{s}(t) \leq 1$, then every solution of the problem (2.1), (2.2) is oscillatory in $G$.

Example 3.1: Consider the system of parabolic differential equations 


$$
\left\{\begin{array}{c}
\frac{\partial}{\partial t}\left[u_{1}(x, t)-u_{1}(x, t-\pi)\right]=\Delta u_{1}(x, t)+2 \Delta u_{1}\left(x, t-\frac{3 \pi}{2}\right) \\
+\frac{1}{2} \Delta u_{2}\left(x, t-\frac{3 \pi}{2}\right)-\frac{3}{2} u_{1}(x, t) \\
-\int_{-\pi}^{-\frac{\pi}{2}} 3 u_{1}(x, t+\xi) d \xi-\int_{-\pi}^{-\frac{\pi}{2}} u_{2}(x, t+\xi) d \xi \\
\frac{\partial}{\partial t}\left[u_{2}(x, t)-u_{2}(x, t-\pi)\right]=2 \Delta u_{2}(x, t)-\Delta u_{1}\left(x, t-\frac{3 \pi}{2}\right) \\
+\Delta u_{2}\left(x, t-\frac{3 \pi}{2}\right)-4 u_{2}(x, t) \\
-\int_{-\pi}^{-\frac{\pi}{2}} u_{1}(x, t+\xi) d \xi-\int_{-\pi}^{-\frac{\pi}{2}} 4 u_{2}(x, t+\xi) d \xi \\
(x, t) \in(0, \pi) \times[0, \infty),
\end{array}\right.
$$

with the boundary condition

$$
\frac{\partial}{\partial x} u_{i}(0, t)=\frac{\partial}{\partial x} u_{i}(\pi, t)=0, t \geq 0, i=1,2
$$

Here $n=1, m=2, r=1, d=1, l=1, \lambda_{1}(t)=1, \rho_{1}=\pi, a_{1}(t)=1, a_{111}(t)=2$, $a_{121}(t)=\frac{1}{2}, \tau_{1}=\frac{3 \pi}{2}, p_{1}(x, t)=\frac{3}{2}, q_{111}(x, t, \xi)=3, q_{121}(x, t, \xi)=1, g_{1}(t, \xi)=t+\xi$, $a_{2}(t)=2, a_{211}(t)=\frac{1}{2}, a_{221}(t)=1, p_{2}(x, t)=4, q_{211}(x, t, \xi)=1, q_{221}(x, t, \xi)=4$, $a=-\pi, b=-\frac{\pi}{2}$. It is easy to see that $Q_{1}(t, \xi)=2, \eta_{1}(t, \xi)=t+\frac{\xi}{2}, t>\frac{3 \pi}{2}$,

$$
\lim _{t \rightarrow \infty} \inf \int_{g_{1}(t, b)}^{t} \int_{a}^{b} Q_{1}(s, \xi) d \xi d s=\lim _{t \rightarrow \infty} \inf \int_{t-\frac{\pi}{2}}^{t} \int_{-\pi}^{-\frac{\pi}{2}} 2 d \xi d s=\frac{\pi^{2}}{2}>\frac{1}{e},
$$

and

$$
\lim _{t \rightarrow \infty} \inf \int_{\eta_{1}(t, b)}^{t} \int_{a}^{b} Q_{1}(s, \xi) d \xi d s=\lim _{t \rightarrow \infty} \inf \int_{t-\frac{\pi}{4}}^{t} \int_{-\pi}^{-\frac{\pi}{2}} 2 d \xi d s=\frac{\pi^{2}}{4}>0 .
$$

Hence all the conditions of Theorem 3.3 are fulfilled. Then every solution of problem (3.12), (3.13) is oscillatory in $(0, \pi) \times[0, \infty)$. In fact, such a solution is $u_{1}(x, t)=\cos x \sin t$, $u_{2}(x, t)=\cos x \cos t$.

\section{Oscillation of the Problem (2.1), (2.3)}

In the domain $\Omega$, we consider the following Dirichlet problem

$$
\left\{\begin{array}{c}
\Delta \omega(x)+\alpha \omega(x)=0 \text { in } \Omega \\
\omega(x)=0 \text { on } \partial \Omega
\end{array}\right.
$$

where $\alpha$ is a constant. It is well known that the least eigenvalue $\alpha_{0}$ of problem (4.1) is positive and the corresponding eigenfunction $\varphi(x)$ is positive on $\Omega$.

Theorem 4.1: If the differential inequality 


$$
\begin{gathered}
{\left[V(t)-\sum_{s=1}^{r} \lambda_{s}(t) V\left(t-\rho_{s}\right)\right]^{\prime}+\alpha_{0} \sum_{j=1}^{d} A_{j}(t) V\left(t-\tau_{j}\right)+p(t) V(t)} \\
+\sum_{h=1}^{l} \int_{a}^{b} Q_{h}(t, \xi) V\left(g_{h}(t, \xi)\right) d \sigma(\xi) \leq 0,
\end{gathered}
$$

has no eventually positive solution, then every solution of problem (2.1), (2.3) is oscillatory in $G$.

Proof: Suppose to the contrary that there is a nonoscillatory solution $u(x, t)=$ $\left\{u_{1}(x, t), u_{2}(x, t), \ldots, u_{m}(x, t)\right\}^{T}$ of problem (2.1), (2.3). We assume that $\left|u_{i}(x, t)\right|>0$ for $t \geq t_{0} \geq 0, i=1,2, \ldots, m$. Let $\delta_{i}=\operatorname{sgn} u_{i}(x, t), Z_{i}(x, t)=\delta_{i} u_{i}(x, t)$, then $Z_{i}(x, t)>0$, $(x, t) \in \Omega \times\left[t_{0}, \infty\right), i=1,2, \ldots, m$. From (H5) and (H7), there exists a number $t_{1} \geq t_{0}$ such that $Z_{i}(x, t)>0, Z_{i}\left(x, t-\rho_{s}\right)>0, Z_{i}\left(x, t-\tau_{j}\right)>0$ and $Z_{i}\left(x, g_{h}(t, \xi)\right)>0$ in $\Omega \times\left[t_{1}, \infty\right)$, $i=1,2, \ldots, m ; s=1,2, \ldots, r ; j=1,2, \ldots, d ; h=1,2, \ldots, l$.

Multiplying both sides of (2.1) by $\varphi(x)$ and integrating with respect to $x$ over the domain $\Omega$, we obtain

$$
\begin{gathered}
\frac{d}{d t}\left[\int_{\Omega} u_{i}(x, t) \varphi(x) d x-\sum_{s=1}^{r} \lambda_{s}(t) \int_{\Omega} u_{i}\left(x, t-\rho_{s}\right) \varphi(x) d x\right]=a_{i}(t) \int_{\Omega} \Delta u_{i}(x, t) \varphi(x) d x \\
+\sum_{k=1}^{m} \sum_{j=1}^{d} a_{i k j}(t) \int_{\Omega} \Delta u_{k}\left(x, t-\tau_{j}\right) \varphi(x) d x-\int_{\Omega} p_{i}(x, t) u_{i}(x, t) \varphi(x) d x \\
\quad-\sum_{k=1}^{m} \sum_{h=1}^{l} \int_{\Omega} \int_{a}^{b} q_{i k h}(x, t, \xi) u_{k}\left(x, g_{h}(t, \xi)\right) \varphi(x) d \sigma(\xi) d x
\end{gathered}
$$

where $t \geq t_{1}, i=1,2, \ldots, m$.

Therefore, we have

$$
\begin{gathered}
\frac{d}{d t}\left[\int_{\Omega} Z_{i}(x, t) \varphi(x) d x-\sum_{s=1}^{r} \lambda_{s}(t) \int_{\Omega} Z_{i}\left(x, t-\rho_{s}\right) \varphi(x) d x\right]=a_{i}(t) \int_{\Omega} \Delta Z_{i}(x, t) \varphi(x) d x \\
+\sum_{j=1}^{d} a_{i i j}(t) \int_{\Omega} \Delta Z_{i}\left(x, t-\tau_{j}\right) \varphi(x) d x+\sum_{k=1, k \neq i}^{m} \sum_{j=1}^{d} a_{i k j}(t) \frac{\delta_{k}}{\delta_{i}} \int_{\Omega} \Delta Z_{k}\left(x, t-\tau_{j}\right) \varphi(x) d x \\
-\int_{\Omega} p_{i}(x, t) Z_{i}(x, t) \varphi(x) d x-\sum_{h=1}^{l} \int_{a}^{b} \int_{\Omega} q_{i i h}(x, t, \xi) Z_{i}\left(x, g_{h}(t, \xi)\right) \varphi(x) d x d \sigma(\xi) \\
-\sum_{h=1}^{l} \sum_{k=1, k \neq i}^{m} \frac{\delta_{k}}{\delta_{i}} \int_{a}^{b} \int_{\Omega} q_{i k h}(x, t, \xi) Z_{k}\left(x, g_{h}(t, \xi)\right) \varphi(x) d x d \sigma(\xi)
\end{gathered}
$$


where $t \geq t_{1}, i=1,2, \ldots, m$.

Using Green's formula and (2.3), we have

$$
\int_{\Omega} \Delta Z_{i}(x, t) \varphi(x) d x=\int_{\Omega} Z_{i}(x, t) \Delta \varphi(x) d x=-\alpha_{0} \int_{\Omega} Z_{i}(x, t) \varphi(x) d x \leq 0,
$$

and

$$
\begin{gathered}
\int_{\Omega} \Delta Z_{k}\left(x, t-\tau_{j}\right) \varphi(x) d x=\int_{\Omega} Z_{k}\left(x, t-\tau_{j}\right) \Delta \varphi(x) d x \\
=-\alpha_{0} \int_{\Omega} Z_{k}\left(x, t-\tau_{j}\right) \varphi(x) d x,
\end{gathered}
$$

where $t \geq t_{1}, k=1,2, \ldots, m ; j=1,2, \ldots, d$.

It follows from (4.4)-(4.6) that

$$
\begin{gathered}
\frac{d}{d t}\left[\int_{\Omega} Z_{i}(x, t) \varphi(x) d x-\sum_{s=1}^{r} \lambda_{s}(t) \int_{\Omega} Z_{i}\left(x, t-\rho_{s}\right) \varphi(x) d x\right] \\
\leq-\alpha_{0} \sum_{j=1}^{d} a_{i i j}(t) \int_{\Omega} Z_{i}\left(x, t-\tau_{j}\right) \varphi(x) d x \\
+\alpha_{0} \sum_{j=1}^{d} \sum_{k=1, k \neq i}^{m}\left|a_{i k j}(t)\right| \int_{\Omega} Z_{k}\left(x, t-\tau_{j}\right) \varphi(x) d x-p_{i}(t) \int_{\Omega} Z_{i}(x, t) \varphi(x) d x \\
+\sum_{h=1}^{l} \sum_{k=1, k \neq i}^{m} \int_{a}^{l} \sum_{h=1}^{b} \int_{a}^{b} q_{i i h}(t, \xi) \int_{\Omega} Z_{i}\left(x, g_{h}(t, \xi)\right) \varphi(x) d x d \sigma(\xi) \\
-\xi \int_{\Omega} Z_{k}\left(x, g_{h}(t, \xi)\right) \varphi(x) d x d \sigma(\xi),
\end{gathered}
$$

where $t \geq t_{1}, i=1,2, \ldots, m$.

Setting

we have

$$
V_{i}(t)=\int_{\Omega} Z_{i}(x, t) \varphi(x) d x, t \geq t_{1}, i=1,2, \ldots, m
$$

$$
\left[V_{i}(t)-\sum_{s=1}^{r} \lambda_{s}(t) V_{i}\left(t-\rho_{s}\right)\right]^{\prime}+\alpha_{0} \sum_{j=1}^{d} a_{i i j}(t) V_{i}\left(t-\tau_{j}\right)
$$




$$
\begin{gathered}
-\alpha_{0} \sum_{j=1}^{d} \sum_{k=1, k \neq i}^{m}\left|a_{i k j}(t)\right| V_{k}\left(t-\tau_{j}\right) \\
+p_{i}(t) V_{i}(t)+\sum_{h=1}^{l} \int_{a}^{b} q_{i i h}(t, \xi) V_{i}\left(g_{h}(t, \xi)\right) d \sigma(x) \\
-\sum_{h=1}^{l} \sum_{k=1, k \neq i}^{m} \int_{a}^{b} \bar{q}_{i k h}(t, \xi) V_{k}\left(g_{h}(t, \xi)\right) d \sigma(\xi) \leq 0,
\end{gathered}
$$

where $t \geq t_{1}, i=1,2, \ldots, m$.

Let $V(t)=\sum_{i=1}^{m} V_{i}(t)$ for $t \geq t_{1}$. It follows from (4.8) that

$$
\begin{gathered}
{\left[V(t)-\sum_{s=1}^{r} \lambda_{s}(t) V\left(t-\rho_{s}\right)\right]^{\prime}} \\
+\alpha_{0} \sum_{j=1}^{d}\left\{\sum_{i=1}^{m}\left[a_{i i j}(t) V_{i}\left(t-\tau_{j}\right)-\sum_{k=1, k \neq i}^{m}\left|a_{i k j}(t)\right| V_{k}\left(t-\tau_{j}\right)\right]\right\} \\
+p(t) V(t)+\sum_{h=1}^{l}\left\{\sum _ { i = 1 } ^ { m } \int _ { a } ^ { b } \left[q_{i h}(t, \xi) V_{i}\left(g_{h}(t, \xi)\right) d \sigma(\xi)\right.\right. \\
\left.\left.\quad-\sum_{k=1, k \neq i}^{m} \int_{a}^{b} \bar{q}_{i k h}(t, \xi) V_{k}\left(g_{h}(t, \xi)\right) d \sigma(x)\right]\right\} \leq 0, t \geq t_{1} .
\end{gathered}
$$

As in the proof of Theorem 3.1, with (4.9), we obtain

$$
\begin{gathered}
{\left[V(t)-\sum_{s=1}^{r} \lambda_{s}(t) V\left(t-\rho_{s}\right)\right]^{\prime}+\alpha_{0} \sum_{j=1}^{d} A_{j}(t) V\left(t-\tau_{j}\right)+p(t) V(t)} \\
+\sum_{h=1}^{l} \int_{a}^{b} Q_{h}(t, \xi) V\left(g_{h}(t, \xi)\right) d \sigma(\xi) \leq 0, t \geq t_{1}
\end{gathered}
$$

which shows that $V(t)=\sum_{i=1}^{m} V_{i}(t)>0$ is a positive solution of the inequality (4.2). This is a contradiction.

By using Theorem 4.1, we have the following theorems.

Theorem 4.2: If all conditions of Theorem 3.1 hold, then every solution of problem (2.1), (2.3) is oscillatory in $G$.

Theorem 4.3: If all conditions of Theorem 3.2 hold, then every solution of problem (2.1), (2.3) is oscillatory in $G$. 
Theorem 4.4: If all conditions of Theorem 3.3 hold, then every solution of problem (2.1), (2.3) is oscillatory in $G$.

Example 4.1: Consider the system of parabolic differential equations

$$
\left\{\begin{array}{c}
\frac{\partial}{\partial t}\left[u_{1}(x, t)-u_{1}(x, t-\pi)\right]=\Delta u_{1}(x, t)+\Delta u_{1}\left(x, t-\frac{3 \pi}{2}\right) \\
-\Delta u_{2}\left(x, t-\frac{3 \pi}{2}\right)-7 u_{1}(x, t) \\
-\int_{-\pi}^{-\frac{\pi}{2}} 5 u_{1}(x, t+\xi) d \xi-\int_{-\pi}^{-\frac{\pi}{2}} 2 u_{2}(x, t+\xi) d \xi \\
\frac{\partial}{\partial t}\left[u_{2}(x, t)-u_{2}(x, t-\pi)\right]=\frac{3}{2} \Delta u_{2}(x, t)+\frac{1}{2} \Delta u_{1}\left(x, t-\frac{3 \pi}{2}\right) \\
+\int_{-\pi}^{-\frac{\pi}{2}} u_{1}(x, t+\xi) d \xi-\int_{-\pi}^{2} 3 u_{2}(x, t+\xi) d \xi \\
(x, t) \in(0, \pi) \times[0, \infty),
\end{array}\right.
$$

with boundary condition

$$
u_{i}(0, t)=u_{i}(\pi, t)=0, t \geq 0, i=1,2 .
$$

Here $n=1, m=2, r=1, d=1, l=1, \lambda_{1}(t)=1, \rho_{1}=\pi, a_{1}(t)=1, a_{111}(t)=1$, $a_{121}(t)=-1, \tau_{1}=\frac{3 \pi}{2}, \quad p_{1}(x, t)=7, \quad q_{111}(x, t, \xi)=5, \quad q_{121}(x, t, \xi)=2, \quad g_{1}(t, \xi)=t+\xi$, $a_{2}(t)=\frac{3}{2}, a_{211}(t)=\frac{1}{2}, a_{221}(t)=2, p_{2}(x, t)=1, q_{211}(x, t, \xi)=1, q_{221}(x, t, \xi)=3$,

$a=-\pi, b=-\frac{\pi}{2}$. It is easy to see that all the conditions of Theorem 4.4 are met. Thus, all the solutions of the problem (4.10), (4.11) are oscillatory in $(0, \pi) \times[0, \infty)$. For instance, $u_{1}(x, t)=\sin x \cos t, u_{2}(x, t)=\sin x \sin t$ is such a solution.

\section{References}

[1] Bainov, D., Cui, B.T., and Minchev, E., Forced oscillation of solutions of certain hyperbolic equations of neutral type, J. Comput. Appl. Math. 72 (1996), 309-318.

[2] Cui, B.T., Yu, Y.H., and Lin, S.Z., Oscillation of solutions of delay hyperbolic differential equations, Acta Math. Appl. Sinica 19 (1996), 80-88. [in Chinese]

[3] Debnath, L. and Li, W.N., Necessary and sufficient conditions for oscillation of delay parabolic equations with variable coefficients, Int. J. Appl. Math. 4 (2000), 133-140.

[4] Fu, X.L. and Zhang, L.Q., The properties of solutions for a class of neutral delay differential inequalities and its applications, Math. Appl. 10:3 (1997), 10-14. [in Chinese]

[5] Fu, X.L. and Zhuang, W., Oscillation of certain neutral delay parabolic equations, $J$. Math. Anal. Appl. 191 (1995), 473-489.

[6] Li, W.N. and Cui, B.T., Oscillation of solutions of neutral partial functional differential equations, J. Math. Anal. Appl. 234 (1999), 123-146.

[7] Li, W.N. and Debnath, L., Oscillation of a system of delay hyperbolic differential equations, Int. J. Appl. Math. 2 (2000), 417-431. 
[8] Mishev, D.P. and Bainov, D.D., Oscillation of the solutions of parabolic differential equations of neutral type, Appl. Math. Comput. 28 (1988), 97-111.

[9] Wiener, J. and Debnath, L., The Fourier method for partial differential equations with piecewise delays, Contemporary Mathematics (ed. by J.R. Graef and J.K. Hale), 129 (1992), 241-263.

[10] Wiener, J. and Debnath, L., Partial differential equations with piecewise constant delay, Intern. J. Math. Math. Sci. 14 (1991), 485-496.

[11] Wiener, J. and Debnath, L., A wave equation with discontinuous time delay, Intern. J. Math. Math. Sci. 15 (1992), 781-788.

[12] Wiener, J. and Debnath, L., Boundary value problems for the diffusion equation with piecewise continuous time delay, Intern. J. Math. Math. Sci. 20 (1997), 187-195.

[13] Wiener, J. and Heller, W., Oscillatory and periodic solutions to a diffusion equation of neutral type, Intern. J. Math. Math. Sci. 22 (1999), 313-348. 


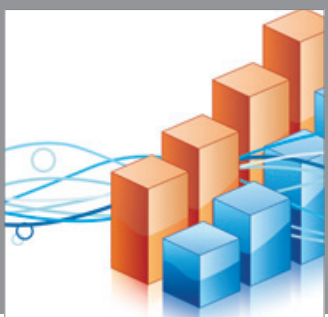

Advances in

Operations Research

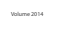

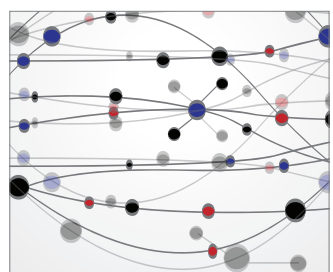

\section{The Scientific} World Journal
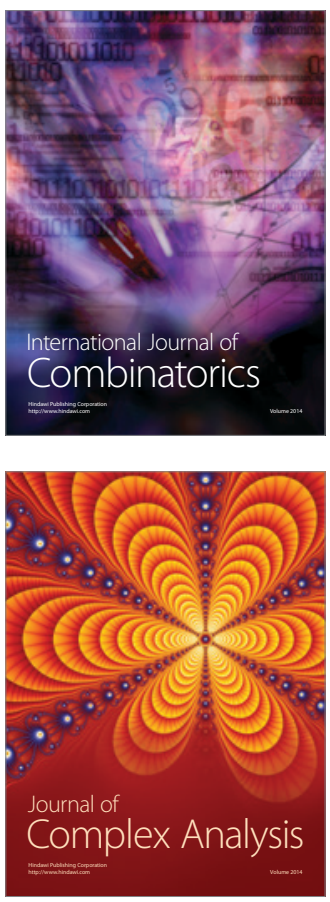

International Journal of

Mathematics and

Mathematical

Sciences
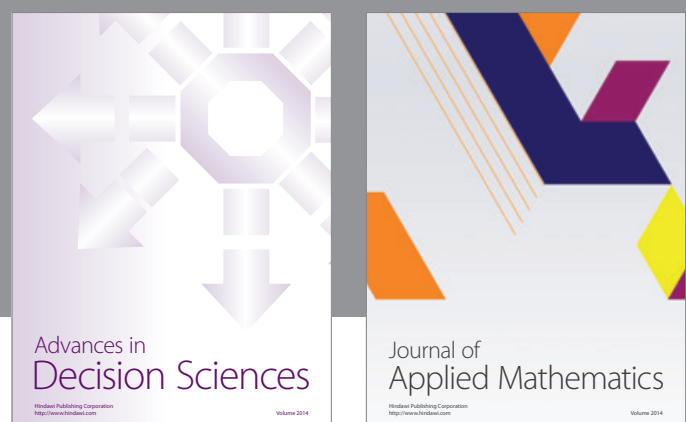

Journal of

Applied Mathematics
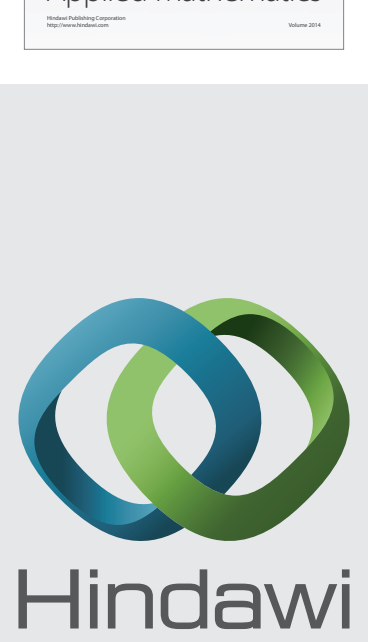

Submit your manuscripts at http://www.hindawi.com
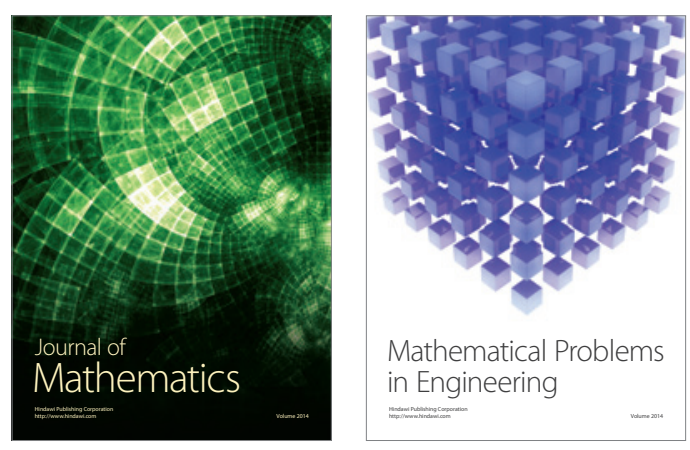

Mathematical Problems in Engineering
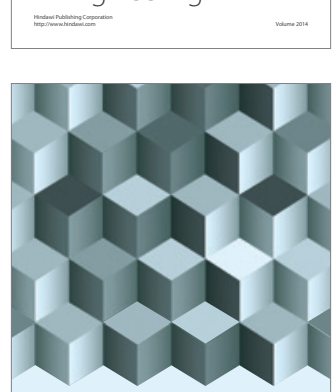

Journal of

Function Spaces
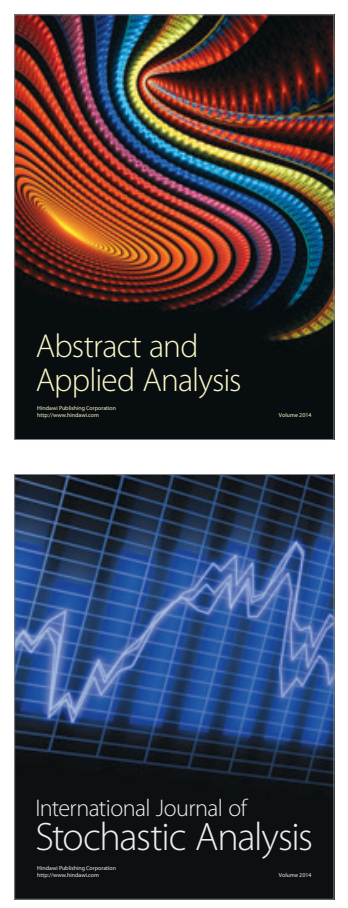

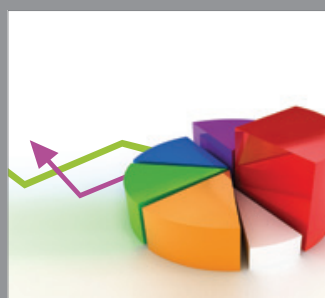

ournal of

Probability and Statistics

Promensencen
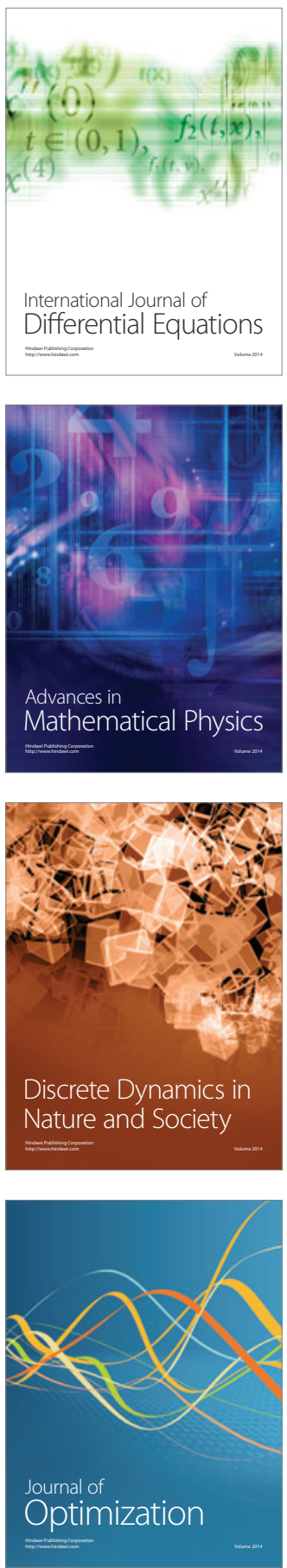